\title{
Author Index to 13th Annual Meeting
}

\author{
Numbers indicate abstract numbers
}

Åkerblom, H. K. 28 Akesode, F. A. 14

Altschuler, N. 32

Anagnostakis, D. 48

Andersen, H. J. 20

Arendt, J. 15

Assa, S. 4. 58

Aubert, M. L. 3

Audi, L. 5

Augier, D. 31

Ballowitz, L. 56

Baron, M. C. 7

Baumann, J. B. 38, 51

Bayard, F. 8, 31

Beaton, G. R. 22, 45

Beaudry, M. 16

Becker, D. 45

Bergadá, C. 32

Bergstrand, C. G. 63

Bernasconi, S. 46

Bewley, T. A. 3

Bidlingmaier, F. 6

Bierich, J. R. 33

Binet, E. 12

Boltzmann, L. 47

Brook, C. G. D. 35

Brunel, R. 47

Butenandt, O. 6

Camus, M. 30

Catterina, A. 50

Cavagni, G. 50

Chaidas, S. 37

Chaussain, J. L. 12

Chiotis, D. 48

Christensen, N. J. 29

Colle, E. 53

Collu, R. 11

Constantinidis, M. 37, 48

Cullen, M. 32

Dacou-Voutetakis, C. 37,48

Darby, C. 49

David, L. 9, 26

David, M. 9

Dazord, A. 5

Delange, F. 30

DeLemater, P. 16

Desbuquois, B. 2

Dickerman, Z. 41

Donnadieu, M. 18

Doret, A.-M. 10

Doron, M. 54

Du Caju, M. V. L. 2, 19, 21

Ducharme, J. R. 11

Dutau, G. 31

Eichner, M. 44

Eshet, R. 4
Ferrandez, A. 34

Fisher, D. A. 16

Forsyth, C. C. 60

François, R. 26

Gilbert, M. 17

Gilet, P. 9

Giovannelli, G. 50

Girard, F. 7, 18

Girard, J. 38, 51

Gleispach, H. 52

Grossman, D. 62

Grumbach, M. M. 3

Gupta, D. 25, 44

Guyda, H. 53

Haberfellner, H. 52

Hagenäs, L. 24

Halabe, E. 41

Heinonen, E. 27

Heinrich, V. 19

Helge, H. 39

Hoogerbrugge, C. 19

Iglesias, J. 59

Illig, R. 13, 46

Iorcansky, S. 32

Jacobsen, B. B. 29

Job, J. C. 12

Josefsberg, Z. 54

Jost, A. 17

Kaplan, S. L. 3

Karp, M. 54

Kastrup, K. W. 20, 29

Kauli, R. 58

Keret, R. 41

Kervran, A. 17

Klemm, W. 25

Knorr, D. 6

Koivukangas, T. 28

Krawczynska, H. 13

Krohn. K. 27

Laron. Z. 4, 41, 54, 58

Lassare, C. 18

Laurance, B. M. 49

Lebacq, E. 26

Leboeuf, G. 11

Lenko, H. L. 55

Letarte, J. 11

Levine, L. S. 43

Liebich, H. M. 25

Limal, J. M. 8

Macabeo, V. 26

Maclaren, N. 14

MacLeod, P. 53

Mäenpää, J. 28
Maroteaux, P. 23

Muihlendahl, K. E. v. 39,56

Mürset, G. 34, 35

Nars, P. W. 38,51

New, M. I. 43

Nielsen, M. D. 57

Nilsson, K. O. 63

Oh, W. 16

Olin, P. 36, 61

Pappo, A. 7

Parth, K. 47

Paunier, L. 10, 15

Pelkonen, R. 27

Perheentupa, J. 27, 28, 55

Pertzelan, A. 58

Petersen, K. E. 57

Peterson, R. E. 43

Phan-Huu-Trung, M. T. 7

Pimstone, B. L. 45

Pluznik, S. 34

Postel-Vinay, M. C. 1

Potau, N. 59

Prader, A. 13, 34, 35

Prager-Lewin, R. 41

Prévòt, C. 21

Puttkamer, K. v. 33

Rager, K. 44

Raiti, S. 14

Rappaport, R. 8, 21

Raux, M. C. 7

Ritzén, M. 24

Rochiccioli, P. 31

Roger, M. 40

Sack, J. 16

Saenger, P. H. 43

Saez, J. M. 5

Sauleda, J. 59

Savage, S. C. L. 60

Schimpff, R. M. 18

Schnakenburg, K. v. 6

Scholler, R. 40

Schönberg, D. 33

Singh, V. 22

Sizonenko, P. C. 10, 15

Snellman, K. 61

Söderholm, A. L. 55

Stanescu, V. 23

Staub, J. J. 38

Suchowerskyi, M. 33

Svan, H. 24

Taipale, V. 55

Tatò, L. 21

Tietze, H. U. 62 
Torresani, T. 46

Toublanc, J. E. 40

Van den Brande, J. L. 19

Vanderschueren-Lodeweyckx, M. 49

Van Der Werff Ten Bosch, J. J. 42

van Male, A. 19

van Steirteghem, A. C. 19
Vassal, J. 12

Vicens-Calvet, E. 59

Wagner, W. 6

Weber, B. 39

Willmann, W. 44

Willner, S. 63

Wolter, R. 19
Wyss, M. 10

Xypolita, A. 37

Zabransky, S. 39

Zachmann, M. 34, 35

Zannos-Mariolea, L. 37

Zimprich, H. 47 